\title{
The opinions of adults about the ban on cigarette sales to minors
}

\author{
H. Ozcebe ${ }^{1}$, N. Bilir ${ }^{1}$, E. Inal $2^{*}$, H. Unlu ${ }^{1}$ E. Beser ${ }^{3}$, G. Can ${ }^{4}$, E. D. Evci Kiraz ${ }^{3}$, P. Okyay ${ }^{3}$, D. Arslantas ${ }^{5}$, F. Abacigil ${ }^{3}$, \\ V. Senol' ${ }^{6}$ E. Turhan? ${ }^{7}$ S. Gokgoz ${ }^{8}$, E. O. Calıkoglu ${ }^{9}$ and Z. Kocan ${ }^{9}$
}

\begin{abstract}
Background: Selling of tobacco products to minors has been banned since 1996 by the tobacco control law in Turkey. However, it is also important for the public to support practices that prevent the access of tobacco products to minors. In addition, every individual has the responsibility of carrying out society based programs that restrict access to tobacco products especially to children and the youths. Social sensitivity is considered an important factor in the prevention of tobacco use. This study aims to learn about the opinions and attitudes of adults with regards to minors access to tobacco products.

Methods: The study was a descriptive study conducted in nine city centers in Turkey. The total number of participants reached was 3241. The questionnaire was developed by the research team and consisted of 22 questions concerning knowledge and behaviors of adults on restriction of tobacco sales to minors and their observations with regards tobacco sales to minors. Data was collected through face to face interview. Pearson chi-square test was used for the bivariate analysis whereas logistic regression was investigate the relationship between "the participant's response against tobacco sales to minors" and the following explanatory variables; "age", "educational status", "income level", "working status", "minors access to cigarettes", "smoking ratio in high school" and "sales of tobacco to minors".
\end{abstract}

Results: More than half of the participants (60.5\%) belonged to the age group 25-44 years, $61.3 \%$ graduated from high school or university. Most of the participants were smoker (39.2\%) or ex-smoker (19.1\%), and $41.7 \%$ of the participants was non-smoker. A greater proportion of the participants (76.2\%) believed that smoking prevalence was greater than $40 \%$ among high school students. One in four (27.8\%) adults did not know that tobacco control law bans sell of tobacco products to minors in Turkey. More than half of the participants (57.1\%) ever witnessed tobacco sales to minors and $63.6 \%$ of them did not act when confronted with the event. Almost all (96.8\%) of the respondents thought that access of minors to tobacco products was not difficult. The results of logistic regression of participant's response against tobacco sales to minor and related factors for current smokers showed that respondents who believed smoking ratio in high school was 4-5 adolescent out of 10 (aOR: 1.59; 95\% Cl: 1.09-2.34) were more likely to give a warning or informing the police or other people as compared to respondents whose perception on the smoking ratio among high school students was 6-7 adolescents out of 10. The results of logistic regression of non-smokers' response against tobacco sales to minor were who are from higher educational level, higher economic status, working status and who believed smoking ratio in high school was 4-5 adolescent out of 10 and 2-3 adolescent out of 10 were more likely to give a warning or informing the police or other people as compared to the others.

(Continued on next page)

\footnotetext{
* Correspondence: ebruinal34@hotmail.com; ebru.inal@hacettepe.edu.tr

${ }^{2}$ Yalova Vocational High School, University of Yalova, Yalova, Turkey

Full list of author information is available at the end of the article
}

(c) The Author(s). 2016 Open Access This article is distributed under the terms of the Creative Commons Attribution 4.0 International License (http://creativecommons.org/licenses/by/4.0/), which permits unrestricted use, distribution, and reproduction in any medium, provided you give appropriate credit to the original author(s) and the source, provide a link to the Creative Commons license, and indicate if changes were made. The Creative Commons Public Domain Dedication waiver (http://creativecommons.org/publicdomain/zero/1.0/) applies to the data made available in this article, unless otherwise stated. 
(Continued from previous page)

Conclusions: Although laws prohibiting the sale of tobacco products to the under age group is very important with regards to accessibility of minors to tobacco products, most of the study participants believed that minors can still easily access tobacco products, and more than half of the participants did not act when confronted with the event. The education, information and monitoring program most especially as it concerns salesman, should be reviewed and strengthened to obey the rules on sales of tobacco products to minors. Education program should be carried out to increase the knowledge and awareness of the community for sale of tobacco to minors. Social sensitivity is important for the prevention of tobacco use and every individual have a responsibility in carrying out this society based program, most especially as it related to prevention of tobacco usage among children and youths.

Keywords: Children, Tobacco, Tobacco point of sale, Turkey

\section{Background}

Tobacco use is one of the most important public health concerns worldwide. The rapid increase in health problems associated with tobacco usage have drawn a lot of attention, and great efforts have been put in place to reduce tobacco use [1].

Tobacco control activities first started in developed countries, and later found their place in all countries' agenda with the World Health Organization (WHO) Framework Convention on Tobacco Control (FCTC) which was accepted at the World Health Assembly in 2003 [2]. FCTC points out two main groups of approaches to reduce tobacco use; the first approach is to reduce the demand for tobacco products, whereas, the second one is to reduce the supply of tobacco products, including placing ban on the sale of tobacco products to children and youth [3, 4]. According to several studies, increased enforcement of laws that ban tobacco sales to minors can reduce access of minors to tobacco products [5-7]. In an observational study on the impact of anti-smoking legislation in Woodridge, a suburban community of Chicago, it was found that both merchant sales and adolescent smoking behavior were reduced after the passage of the law [6]. According to the study, the rates of cigarette experimentation and regular use of cigarettes by adolescents were reduced by over $50 \%$ between the pre- and post-test observation periods. In addition, the reduction in cigarettes use was still apparent after 7 years [6]. Though previous studies suggest that the enforcement of laws banning the sale of tobacco products to the underage can lead to a reduction in tobacco use among minors, by making it harder to purchase cigarettes [7]. However, the effectiveness of tobacco control laws is undermined by other "social sources" of cigarettes [8]. For example, an intervention study done by Forster and colleagues to enact local ordinances, change retail merchants' behavior, and promote enforcement of laws prohibiting illegal sales to minors in 14 Minnesota communities found that the prevalence of smoking among the young age groups was much less in the intervention communities as compared to other communities. In addition, they also observed that youths who reported ever smoking were very likely to cite social sources for cigarettes, though, older youth and those reporting weekly smoking also reported purchasing their own tobacco [5]. There are also similar studies showing that tobacco laws alone are not enough to reduce illegal sales to minors [8-10].

Public support for practices on the prevention of access to tobacco products for minors is very important if any progress is to be made. Studies have been done to this effect [11-13]. A 1992 national telephone poll of 1200 adults residing in New York showed that approximately $83 \%$ were in favor of legislations banning tobacco advertisements targeted at teenagers in New York. In addition, three quarters of smokers also supported a ban on tobacco advertisements targeted at teenagers [14]. Another study shows that most of the American public do not believe that laws prohibiting the sale of tobacco to minors are adequately enforced [15]. In a survey conducted in the United States, it was reported that 8 out of 10 adults felt it was "very easy" or "somewhat easy" for teenagers to buy cigarettes near where they live [16]. Some literatures suggest that majority of adults think laws banning the sale of tobacco products to minors have not been adequately enforced $[15,17,18]$.

Tobacco control activities first started in Turkey through the Law on Prevention of Harms of Tobacco Use in 1996 [19]. This Law was further amended in 2008, and played a role in making Turkey the third country in the world to be $100 \%$ smoke-free country [20]. The first and the amended law banned selling of tobacco products to minors less than 18 years of age $[19,21]$. Even though there are some difficulties in the implementation of this item, Turkey has recorded important achievements in tobacco control and is the first and single country in the world implementing all six strategies of MPOWER with success [22]. However, tobacco use is still considered to be one of the main public health issues in Turkey, as Turkey is one of the countries with the highest tobacco usage rate in the world [22]. 
The frequency of smoking is $27.1 \%$ among older than 15 years in Turkey and about 15 million people use cigarette and other tobacco products [22]. According to the Global Adult Tobacco Survey (GATS), one fifth of smoker adults start using tobacco products before 15 years and more than half of adults start using tobacco products before 18 years [22]. This result reveals that tobacco use is quite prevalent among the young, also, a great number of smokers starts this behavior during their youth. Thus, it is very important to prevent children and the young from starting tobacco use.

The Turkish Law on the Prevention and Control of Hazards of Tobacco Products, Law No. 4207 has an article about the "prohibition of access to tobacco use for individuals who are under the age of 18 through sale and distribution" [19]. This is a very important law with regards to tobacco control as during tobacco control among minors, one of the focus is to prevent minors from accessing tobacco products with emphasis on advertising bans, sponsorship, promotion, brand stretching and regulations aimed at public awareness and point of sales [19]. This study aims to learn about the opinions and attitudes of adults with regards to minors access situations to tobacco products.

\section{Methods}

\section{Study design and data collection}

The study was a descriptive study conducted in nine city centers of Turkey; Ankara, Aydın, Erzurum, Eskisehir, İzmir, Gümüşhane, Kayseri, Kırklareli, and Trabzon. These cities were selected by convenience from different geographic regions of the country. Thus, the study findings are not representative of the whole country.

The study was organized by the Institute of Public Health, University of Hacettepe. Faculty members from the departments of public health or public health specialist in each city coordinated field data collection. The study was funded by Hacettepe University Scientific Research Projects Coordination Unit.

The sample size was determined by accepting the prevalence as 0.50 and error as 0.05 (with 95\% confidence interval). Participation in the study was voluntary and convenience sampling technique was used with the aim of reaching 400 people in each city center. Participant selection criteria included adults aged 18 years and above.

The questionnaire form was developed by the study team, based on previous literatures. The questionnaire consisting of 22 questions regarding smoking behavior of the participants, knowledge of tobacco control law, observation and reactions on tobacco sales to minors. The items in the questionnaire consisted of sociodemographic characteristics of participants (6 questions), questions on individual smoking profile (4 questions), participants opinion about smoking among minors (5 questions), knowledge about prohibition of sales of tobacco to minors (2 questions), ever witnessed tobacco sale to minors and their reactions (3 questions) and suggestion about tobacco sale to minors (2 questions). The questionnaire was initially pretested and data was collected through face to face interview.

The questionnaire was initially pretested and data was collected through face to face interview. All interviewers were trained by the local research team in each city. The interviewers comprised university graduates or final year students. The expected total number of people to be interviewed was 3600 however, the total number of people reached was 3241 .

Variables (i) Socio-demographic variables; Socio demographic variables included age, educational status, working status and income level. Age was recorded into 18-24, 25-34, 35-44, 45-54 and 55 and above years. Educational status was recorded into literate, primary (5 years), secondary (8 years), high school (11 years), university and others. Working status was grouped into working and not working. Income level was grouped into 5 groups: income level was grouped into lowest, lower middle, middle, higher middle and highest.

(ii) Perception and knowledge of tobacco use and sales to minor and tobacco control law covariates; this included questions on smoking ratio in high school, sales of tobacco to minors, penalty for sales of tobacco to minors, the places providing cigarettes to minors, and minors access to cigarettes. Smoking ratio in high school was recorded into the following groups; one adolescent out of 10, 2-3 adolescents out of 10, 4-5 adolescents out of 10 , and 6-7 adolescents out of 10. Sales of tobacco to minors were grouped into prohibited, allowed with their parents and not prohibited. Penalty for sales of tobacco to minors was categorized into fine, cancellation of sales certification, and imprisonment, the places providing cigarettes to minors were grouped into grocery/market, friends, vendor, and family. Minors' access to cigarettes was grouped into very easy, not very difficult and very difficult.

(iii) Ever witnessed tobacco sales to minor and response against the situation variables; Respondents were asked if they witnessed any tobacco sale to minors, the sellers response to minor and the participants response against tobacco sales to minor. Ever witnessed any tobacco sales to minors was grouped into yes and no. The sellers response to minor was categorized into asking his/her ID card and not asking anything and selling tobacco product. The participant's response against this event include the following categories; 
Doing nothing, warning the sellers, warning the owner, warning the child, informing the police, and talking to the other people about this event.

(iv) Smoking status: Smoking status was grouped into current smokers, former smokers and never smoked.

\section{Statistical analysis}

For the study sample characteristics, variables were reported as frequencies and percentages. Pearson chisquare tests were conducted to examine bivariate differences with smoking status where the assumption of the expected count less than 5 should not exceed 20\% for variables was satisfied, if otherwise exact chi-square test was used. "Participant's response against tobacco sales to minor" was specified as dependent variable and dichotomized into warming/informing and nothing (as reference). Additionally, independent variables which includes "age", "educational status", "income level", "minors access to cigarettes" and "sales of tobacco to minors" were grouped as follows: "Age" was classified into 18-24, 25-34 and 35 and above; "educational status" was classified into secondary and below, high school and university; "income level" was classified into middle and below, and higher middle and above; "minors access to cigarettes" was classified into very easy, not very difficult and very difficult and "sales of tobacco to minors" was classified into prohibited and not prohibited, allowed with their parents. Since "smoking status" was thought the most important factor affecting the behavors, two logistic regression models were performed to identify the relationship between "participant's response against tobacco sales to minor" and related factors for current smokers and participants who never smoked. For current smokers, logistic regression model was constructed to identify the relationship between "participant's response against tobacco sales to minor" and the following explanatory variables; socio-demographic variables (age, educational status, income level, working status), "minors access to cigarettes" and "smoking ratio in high school". For participants who never smoked, logistic regression model was constructed to identify the relationship between "participant's response against tobacco sales to minor" and the following explanatory variables; sociodemographic variables (age, educational status, income level, working status), "smoking ratio in high school" and "sales of tobacco to minors". Reference categories were shown in Tables 4 and 5. Hosmer-Lemeshow statistics was used to assess model assumption. The variables which were found as significant according to Pearson chi-square test were included into logistic regression model as independent variables. Since some participants did not answered all the questions, there were some missing observations in the dataset. While performing multiple logistic regression, list wise deletion method was used to handle with missing observations. A $p$-value below 0.05 was accepted as significant.

\section{Ethics}

This study was approved by Non-interventional Clinical Researches Ethics Board of Hacettepe University (GO 13/242-19).

\section{Results}

Most of the participants were smoker (39.2\%) or exsmoker (19.1\%), and $41.7 \%$ of the participants was nonsmoker; $16.1 \%$ of the smokers started smoking daily before the age of 15 while $42.6 \%$ started smoking between ages 15-17 (data is not given in the table).

A greater proportion of respondents (60.5\%) were in the age group 25-44 years age group, approximately $61.3 \%$ of participants were high school or university graduates. A greater percentage of study participants (65.9\%) were currently working. About $85.7 \%$ had middle and below income level. More than half of the participants $(55.5 \%)$ who were current smokers were in the age group 18-34 years. Participants who were never smoked had mostly high school and above educational status (64.0\%), whereas more than half of the participants (64.7\%) who were current smokers had high school and above educational status. Most of the participants who were never smoked, smoked and quit, current smokers had middle income level (43.7, 49.0 and $43.7 \%$ respectively) (Table 1 ).

All the participants believed that smoking behavior was highly prevalent among adolescents. Only $7.2 \%$ of participants believed that one out of 10 high school students were smokers, most of the participants (76.2\%) believed that smoking prevalence was more than $40 \%$ among high school students. Participants who were current smokers thought that more high school students were current smoking as compared to non-smokers $(p=0.001)$ (Table 2).

A higher percentage of the participants believed that it was prohibited to sell tobacco to minors, however, 9.8\% of respondents believed that minors were allowed to buy tobacco products with their parents and $18.0 \%$ thought that there were no laws prohibiting the sale of tobacco to minors. A greater proportion of respondents believed that the penalty for sales of tobacco to minors was fine $(56.4 \%)$ and a greater percentage of current smokers $(55.3 \%)$ as compared to non-smokers believed so $(p=0.003)$ (Table 2).

Almost half of the participants (54.6\%) believed that minors accessed tobacco products from grocery/market or vendors, a greater proportion of current smokers as compared to non-smokers believed that minors accessed cigarettes from vendors (14.8 and 8.7\%) (Table 2). 
Table 1 Some sociodemographic characteristics of participants

\begin{tabular}{|c|c|c|c|c|c|c|c|c|c|}
\hline \multirow{3}{*}{$\begin{array}{l}\text { Socio-demographic } \\
\text { characteristics } \\
\text { Age }\end{array}$} & \multicolumn{6}{|c|}{ Smoking Status } & \multicolumn{2}{|l|}{ Total } & \multirow[t]{3}{*}{$P$} \\
\hline & \multicolumn{2}{|c|}{ Never } & \multicolumn{2}{|c|}{ Smoked and quit } & \multicolumn{2}{|c|}{ Current smoker } & \multirow[t]{2}{*}{$\mathrm{N}$} & \multirow[t]{2}{*}{$\%$} & \\
\hline & $n$ & $\%$ & $n$ & $\%$ & $n$ & $\%$ & & & \\
\hline $18-24$ & 275 & 22.3 & 49 & 8.7 & 187 & 16.0 & 511 & 17.2 & $<0.001$ \\
\hline $25-34$ & 483 & 39.2 & 171 & 30.3 & 462 & 39.5 & 1116 & 37.6 & \\
\hline $35-44$ & 267 & 21.7 & 113 & 20.0 & 299 & 25.5 & 679 & 22.9 & \\
\hline $45-54$ & 138 & 11.2 & 123 & 21.8 & 145 & 12.4 & 406 & 13.7 & \\
\hline 55 and over & 68 & 5.5 & 109 & 19.3 & 78 & 6.7 & 255 & 8.6 & \\
\hline Total & 1231 & & 565 & & 1171 & & 2967 & 100.0 & \\
\hline \multicolumn{10}{|l|}{ Educational Status } \\
\hline Literate & 123 & 9.6 & 45 & 7.8 & 71 & 5.9 & 239 & 7.8 & $<0.001$ \\
\hline Primary (5 years) & 122 & 9.6 & 97 & 16.7 & 164 & 13.7 & 383 & 12.5 & \\
\hline Secondary (8 years) & 214 & 16.8 & 112 & 19.3 & 234 & 19.5 & 560 & 18.3 & \\
\hline High school (11 years) & 345 & 27.0 & 160 & 27.6 & 389 & 32.5 & 894 & 29.3 & \\
\hline University & 453 & 35.5 & 165 & 28.4 & 326 & 27.2 & 944 & 30.9 & \\
\hline Others (vocational school, etc.) & 19 & 1.5 & 1 & 0.2 & 13 & 1.1 & 33 & 1.1 & \\
\hline Total & 1276 & & 580 & & 1197 & & 3053 & 100.0 & \\
\hline \multicolumn{10}{|l|}{ Working Status } \\
\hline Working & 768 & 61.0 & 375 & 65.4 & 847 & 71.7 & 1990 & 66.0 & $<0.001$ \\
\hline Not working & 492 & 39.0 & 198 & 34.6 & 335 & 28.3 & 1025 & 34.0 & \\
\hline Total & 1260 & & 573 & & 1182 & & 3015 & 100.0 & \\
\hline \multicolumn{10}{|l|}{ Income Level } \\
\hline Lowest & 72 & 5.7 & 36 & 6.1 & 60 & 5.0 & 168 & 5.5 & $<0.001$ \\
\hline Lower middle & 486 & 38.4 & 199 & 34.0 & 400 & 33.5 & 1085 & 35.6 & \\
\hline Middle & 553 & 43.7 & 287 & 49.0 & 522 & 43.7 & 1362 & 44.7 & \\
\hline Higher middle & 129 & 10.2 & 52 & 8.9 & 169 & 14.2 & 350 & 11.5 & \\
\hline Highest & 25 & 2.0 & 12 & 2.0 & 43 & 3.6 & 80 & 2.6 & \\
\hline Total & 1265 & & 586 & & 1194 & & 3045 & 100.0 & \\
\hline
\end{tabular}

Note: Some participants did not answer all the questions

Only $3.2 \%$ of the participants believed that minors' access to cigarettes was very difficult. According to smoking status, smokers (66.9\%) believed that minors access to cigarettes was very easy as compared to nonsmokers $(59.3 \%)(p=0.001)$. (Table 2).

More than half of the participants (57.1\%) ever witnessed any tobacco sales to minors, current smokers reported higher percentage $(63.8 \%)$ of having witnessed tobacco sales to minor as compared to nonsmokers $(p<0.001)$ (Table 3).

Approximately $64 \%$ of respondents who ever witnessed any tobacco sales to minors did not act when confronted with the event and current smokers made up a higher proportion of the group "doing nothing" as compared to non-smokers, however, nonsmokers had more positive response with regards to warning/informing the police or other people $(p=0.002)$. Approximately $88.0 \%$ of the salesmen sold tobacco products without asking the child's age or asking for an identity card (Table 3).

Two logistic regression models with multiple predictors were constructed to investigate relationship between participant's response against tobacco sales to minors and related factors for current smokers and participants who never smoked. The results of logistic regression models for current smokers were given in Table 4. The results of logistic regression model showed that the model assumptions were satisfied $(p=0.271)$ and the model was found to be statistically significant $(p<$ 0.001 ). Respondents who believed smoking ratio in high school was 4-5 adolescent out of 10 (aOR: 1.59; 95\% CI: 1.09-2.34), were more likely to give a warning or informing the police or other people as compared to respondents whose perception on the smoking ratio among high school students was 6-7 adolescents out of 10, whereas $2-3$ adolescent out of 10 (aOR: 0.74; 95\% CI: 
Table 2 The perception and knowledge of tobacco use and sales to minor and tobacco control law by smoking status

\begin{tabular}{|c|c|c|c|c|c|c|c|c|c|}
\hline \multirow{3}{*}{$\begin{array}{l}\text { Perception and Knowledge on } \\
\text { Tobacco Use and Sales } \\
\text { The smoking ratio in high school }\end{array}$} & \multicolumn{6}{|c|}{ Smoking Status } & \multicolumn{2}{|l|}{ Total } & \multirow[t]{3}{*}{$P$} \\
\hline & \multicolumn{2}{|l|}{ Never } & \multicolumn{2}{|c|}{ Smoked and quit } & \multicolumn{2}{|c|}{ Current smoker } & \multirow[t]{2}{*}{$\mathrm{N}$} & \multirow[t]{2}{*}{$\%$} & \\
\hline & $n$ & $\%$ & $n$ & $\%$ & $n$ & $\%$ & & & \\
\hline One adolescent out of 10 & 88 & 7.1 & 44 & 7.8 & 80 & 7.0 & 212 & 7.2 & 0.001 \\
\hline $2-3$ adolescents out of 10 & 240 & 19.3 & 90 & 15.9 & 161 & 14.0 & 491 & 16.6 & \\
\hline $4-5$ adolescents out of 10 & 456 & 36.7 & 196 & 34.7 & 385 & 33.5 & 1037 & 35.0 & \\
\hline 6-7 adolescents out of 10 & 460 & 37.0 & 235 & 41.6 & 523 & 45.5 & 1218 & 41.2 & \\
\hline Total & 1244 & & 565 & & 1149 & & 2958 & 100.0 & \\
\hline \multicolumn{10}{|l|}{ Sales of tobacco to minors } \\
\hline Prohibited & 888 & 72.5 & 408 & 72.3 & 837 & 71.8 & 2133 & 72.2 & 0.700 \\
\hline Allowed with their parents & 120 & 9.8 & 61 & 10.8 & 107 & 9.2 & 288 & 9.8 & \\
\hline Not prohibited & 216 & 17.6 & 95 & 16.8 & 221 & 19.0 & 532 & 18.0 & \\
\hline Total & 1224 & & 564 & & 1165 & & 2953 & 100.0 & \\
\hline \multicolumn{10}{|l|}{ Penalty for sales of tobacco to minors } \\
\hline Fine & 565 & 58.7 & 240 & 53.9 & 508 & 55.3 & 1313 & 56.4 & 0.003 \\
\hline Cancellation of sales certification & 256 & 26.6 & 140 & 31.5 & 310 & 33.8 & 706 & 30.4 & \\
\hline Imprisonment & 142 & 14.7 & 65 & 14.6 & 100 & 10.9 & 307 & 13.2 & \\
\hline Total & 963 & & 445 & & 918 & & 2326 & 100.0 & \\
\hline \multicolumn{10}{|c|}{ Major point were cigarettes are provided to minors } \\
\hline Grocery/Market & 554 & 44.7 & 265 & 47.4 & 456 & 40.0 & 1275 & 43.4 & $<0.001$ \\
\hline Friends & 524 & 42.3 & 217 & 38.8 & 454 & 39.8 & 1195 & 40.7 & \\
\hline Vendor & 108 & 8.7 & 52 & 9.3 & 169 & 14.8 & 329 & 11.2 & \\
\hline Family & 52 & 4.2 & 25 & 4.5 & 62 & 5.4 & 139 & 4.7 & \\
\hline Total & 1238 & & 559 & & 1141 & & 2938 & 100.0 & \\
\hline \multicolumn{10}{|l|}{ Minors access to cigarettes } \\
\hline Very easy & 749 & 59.3 & 350 & 60.3 & 795 & 66.9 & 1894 & 62.5 & 0.001 \\
\hline Not very difficult & 477 & 37.8 & 209 & 36.0 & 354 & 29.8 & 1040 & 34.3 & \\
\hline Very difficult & 37 & 2.9 & 21 & 3.6 & 40 & 3.4 & 98 & 3.2 & \\
\hline Total & 1263 & & 580 & & 1189 & & 3032 & 100.0 & \\
\hline
\end{tabular}

Note. Some participants did not answer all the questions

0.40-1.37) were less likely to give a warning or informing the police or other people (Table 4).

The results of logistic regression models for participants who never smoked were given in Table 5. The results of logistic regression model showed that the model assumptions were satisfied $(p=0.215)$ and the model was found to be statistically significant $(p<0.001)$. Respondents who believed smoking ratio in high school was 4-5 adolescent out of 10 (aOR: 1.71 ; 95\% CI: $1.12-$ 2.62), and 2-3 adolescent out of 10 (aOR: 2.25 ; 95\% CI: 1.33-3.83) were more likely to give a warning or informing the police or other people as compared to respondents whose perception on the smoking ratio among high school students was 6-7 adolescents out of 10 . Having knowledge of the prohibition of tobacco sales to minors (aOR: 2.06; 95\% CI: 1.34-3.16) was associated with higher likelihood of giving a warning or informing the police or other people as compared to not having any knowledge on the prohibition of tobacco sales to minors. Participants who had highest or higher middle income level (aOR: 1.86; 95\% CI: 1.28-2.72) were more likely to give a warning or informing the police or other people as compared to respondents who had middle, lower middle or lowest income level. Respondents knowing sales of tobacco products prohibited to minors (aOR: 2,06; 95\% CI: $1.34-3.16$ ) were more likely to give a warming or informing the police or other people as compared to respondents who were not working (Table 5).

\section{Discussion}

Turkey is known as a country with a history of important successes in tobacco control [23]. The prevention of tobacco access is one the most important intervention, 
Table 3 Ever witnessed tobacco sales to minor and response against the situation by smoking status

\begin{tabular}{|c|c|c|c|c|c|c|c|c|c|}
\hline \multirow[t]{3}{*}{ Experience of participants on tobacco sales } & \multicolumn{6}{|c|}{ Smoking Behavior } & \multicolumn{2}{|l|}{ Total } & \multirow[t]{3}{*}{$P$} \\
\hline & \multicolumn{2}{|c|}{ Never } & \multicolumn{2}{|c|}{ Smoked and quit } & \multicolumn{2}{|c|}{ Current smoker } & \multirow[t]{2}{*}{$N$} & \multirow[t]{2}{*}{$\%$} & \\
\hline & $n$ & $\%$ & $\mathrm{n}$ & $\%$ & $\mathrm{n}$ & $\%$ & & & \\
\hline \multicolumn{10}{|l|}{ Ever witnessed any tobacco sales to minors } \\
\hline Yes & 630 & 50.0 & 343 & 59.0 & 758 & 63.8 & 1731 & 57.1 & $<0.001$ \\
\hline No & 631 & 50.0 & 238 & 41.0 & 430 & 36.2 & 1299 & 42.9 & \\
\hline Total & 1261 & & 581 & & 1188 & & 3030 & 100.0 & \\
\hline \multicolumn{10}{|l|}{ The seller's response to the child } \\
\hline Asking his/her identity card & 76 & 12.0 & 51 & 14.8 & 80 & 10.6 & 207 & 12.0 & 0.176 \\
\hline Not asking anything and selling tobacco product & 557 & 88.0 & 294 & 85.2 & 677 & 89.4 & 1510 & 88.0 & \\
\hline Total $^{\mathrm{a}}$ & 633 & & 345 & & 749 & & 1727 & 100 & \\
\hline \multicolumn{10}{|l|}{ The participant's response against tobacco sales to minors } \\
\hline Doing nothing & 372 & 60.4 & 199 & 59.1 & 504 & 68.3 & 1075 & 63.6 & 0.002 \\
\hline Warning the seller's & 98 & 15.9 & 61 & 18.1 & 84 & 11.4 & 243 & 14.4 & \\
\hline Warning the owner & 33 & 5.4 & 14 & 4.2 & 22 & 3.0 & 69 & 4.1 & \\
\hline Warning the child & 77 & 12.5 & 46 & 13.6 & 89 & 12.1 & 212 & 12.5 & \\
\hline Informing the police & 21 & 3.4 & 3 & 0.9 & 14 & 1.9 & 38 & 2.2 & \\
\hline Talking to the other people about this event & 15 & 2.4 & 14 & 4.2 & 25 & 3.4 & 54 & 3.2 & \\
\hline Total $^{a}$ & 616 & & 337 & & 738 & & 1691 & 100.0 & \\
\hline
\end{tabular}

Note: ${ }^{\text {T}}$ The participants were witness at any tobacco sale under 18 years old

thus, preventing children and youth from starting tobacco. The most important stage in the prevention of accessibility is determined as increasing taxes and applications for point of sales [1,2].

In this study, 3241 people were interviewed in 9 city centers. The people interviewed are mainly young adults and their educational status was higher than average level of education when compared to the general population. Most of the smoker and ex-smoker participants declared that they started daily smoking at adolescent ages. The participants in the current study are also aware of this as they believed that smoking is a very common behavior among high school students. According to GATS (2012), among ever daily smokers who were aged 18-34 years, 17.1 years was the average age daily smokers started smoking [22]. These findings actually support that tobacco use is accepted as a public health problem among young people.

As earlier stated, one of the most important intervention for the prevention of tobacco use among youths is the prevention of accessibility. In consonance, previous studies have shown that enforcement of laws against tobacco sales to minors is an effective strategy to maintain a barrier for accessibility of tobacco in the community $[5,6,8]$. WHO FCTC also has some suggestion on the enforcement of tobacco sales to minor through regulations of point of sales [24]. The regulation on tobacco sale to minors in Turkey is old, it had been prohibited since 1996, prison sentence was later added as a new regulation in 2008 [19, 21]. In this study, $72.2 \%$ of the all respondents gave the true answer with regards rule on tobacco sales to minors, there is not any difference of their knowledge on rule of sales of tobacco to minors between smokers and non-smokers. However, the result of our findings shows that prison sentence is less known (13.2\%) and goes to support the fact that information about prison sentence being a part of the law has not become widespread in the community, especially among the smokers. The way of giving information about tobacco selling ban to minors is by having poster at points of sales as well as inspection of this sale points to see if they have the ban posters in the country. However, the posters have only information showing that tobacco sell is banned to children less than 18 years of age. The results of this study revealed that this intervention has been quite effective in increasing the awareness of adults about tobacco selling ban to minors, but this intervention was not effective in increasing the knowledge on pertaining prison sentence. It is worth noting that a high-level debate has been ongoing among the leaders of tobacco control in the country. Some are of the opinion that informative program about the ban of cigarette sale to children under the age of 18 can create a wrong perception as this information can encourage minors to be eager to smoke after the age of 18. Because of this high-level debate, there is not any proactive campaign carried out on sale of tobacco to youth, this is a barrier in front of the distribution of the true information widely in the country. 
Table 4 Logistic regression results of participant's response against tobacco sales to minor and related factors for current smokers

\begin{tabular}{|c|c|c|c|}
\hline & OR & $P$ & $95 \% \mathrm{Cl}$ \\
\hline Constant & 0.15 & $<0.001$ & \\
\hline \multicolumn{4}{|l|}{ Educational Status } \\
\hline Literate/Primary/Secondary (ref) & 1 & & \\
\hline High School & 1.52 & 0.054 & $0.99-2.33$ \\
\hline University & 1.47 & 0.095 & $0.94-2.30$ \\
\hline \multicolumn{4}{|l|}{ Age group } \\
\hline $18-24$ (ref) & 1 & & \\
\hline $25-34$ & 0.97 & 0.914 & $0.55-1.69$ \\
\hline 35 and above years & 1.70 & 0.056 & $0.98-2.95$ \\
\hline \multicolumn{4}{|l|}{ Income level } \\
\hline Middle, lower middle,/ lowest (ref) & 1 & & \\
\hline Highest /higher middle & 1.38 & 0.078 & $0.96-1.96$ \\
\hline \multicolumn{4}{|l|}{ Working Status } \\
\hline Not working (ref) & 1 & & \\
\hline Working & 1.28 & 0.266 & $0.83-1.97$ \\
\hline \multicolumn{4}{|l|}{ Minors access to cigarettes } \\
\hline Very easy/Not very difficult (ref) & 1 & & \\
\hline Very difficult & 3.11 & 0.076 & $0.89-10.89$ \\
\hline \multicolumn{4}{|l|}{ Smoking ratio in high school } \\
\hline 6-7 adolescents out of 10 (ref) & 1 & & \\
\hline $4-5$ adolescents out of 10 & 1.59 & 0.016 & $1.09-2.34$ \\
\hline 2-3 adolescents out of 10 & 0.74 & 0.344 & $0.40-1.37$ \\
\hline One adolescent out of 10 & 1.94 & 0.139 & $0.81-4.67$ \\
\hline
\end{tabular}

Literatures show that enforcement of tobacco sales ban to children decreases accessibility of minors to tobacco products $[5,6,8]$. In this study, $66.7 \%$ of the smokers and $59.3 \%$ of non-smokers think that accessibility of tobacco products is very easy and a larger proportion of respondents assume that individuals who are under the age of 18 can buy tobacco from markets or vendors. Vendors are mentioned by smokers more than non-smokers and ex-smokers $(p<0,001)$. GYTS in Turkey also supports the results of this study, it states that $53.7 \%$ of the youth buy cigarettes from markets and $79.1 \%$ of young smokers are not refused sell [25]. GYTS results are in consonance with our study finding. There are also other supporting studies [15, 26, 27]. According to Elders, laws prohibiting the sale of tobacco to minors are not adequately enforced [15]. Also, Marcus and colleagues in their study found that 8 out of 10 adults believed teenagers can easily reach tobacco products "very easily" or "somewhat easily" in their neighborhood [16]. Enforcement of laws against tobacco sales to minors should be monitored and strengthened at groceries and
Table $\mathbf{5}$ Logistic regression results of participant's response against tobacco sales to minor and related factors for participants who never smoked

\begin{tabular}{llll}
\hline & OR & $P$ & $95 \% \mathrm{Cl}$ \\
\hline Constant & 0.16 & $<0.001$ &
\end{tabular}

Educational Status

Literate/Primary/Secondary (ref) 1

$\begin{array}{llll}\text { High School } & 0.91 & 0.709 & 0.57-1.47\end{array}$

Age

$$
\text { 18-24 (ref) }
$$

25-34

$050.843 \quad 0.56-1.60$

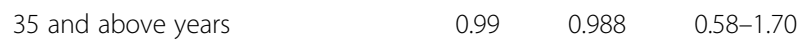

Income level

Middle, lower middle,/ lowest (ref) 1

$\begin{array}{llll}\text { Highest /higher middle } & 1.86 & 0.001 & 1.28-2.72\end{array}$

Working Status

Not working (ref)

Working

$23-0.326-0.81-1.88$

Sales of tobacco to minors

Not prohibited (ref)

Prohibited

$2.06 \quad 0.001 \quad 1.34-3.16$

Smoking ratio in high school

6-7 adolescents out of 10 (ref) 1

4-5 adolescents out of 10

$\begin{array}{llll}\text { One adolescent out of } 10 & 2.06 & 0.105 & 0.86-4.94\end{array}$

Classification Rate: \% 64.5; Hosmer-Lemeshow Test: $p=0.215$

vendors [28]. Changing retail merchants' behavior to prevent illegal sales of tobacco to minors should be enforced and promoted [5]. In Turkey, even though the salesman are informed about the regulation on the sales of tobacco yearly, still children are able to access tobacco products from markets and vendors. As such, in the country, the education, information and monitoring program most especially as it concerns salesman and owners of vendors, should be reviewed and strengthened to obey the rules on sales of tobacco products to minors.

Previous studies have shown that youth can use social sources to access tobacco product [5, 8-11]. Our study findings are in consonance, the result of our study shows that adults are also aware of children accessibility of tobacco products through families and friends.

Public support in the enforcement of laws governing tobacco sales is also important if preventing the access of tobacco product to minors is to be achieved. In our study, $63.8 \%$ of the smokers, $59.0 \%$ of ex-smokers and $50.0 \%$ of non-smokers declared that they witnessed cigarette sale to minors, among all witness participants, 
88.0\% said the sellers' response was not asking anything and selling the tobacco product. Also, most of the participants did not take any action, the percentage of smokers not taking any reaction was much higher than non-smokers. More ex-smokers reacting this event is than smokers and non-smokers, they would have had some health problems because of smoking in the past. In a society where there is a target of preventing the sale of cigarette to minors, the salesman have a role to play, in addition, it is expected that all other individuals in the society should be sensitive and play a role in the monitoring of sale points of tobacco. Increasing the awareness of society in terms of age restrictions with regards tobacco sales will strengthen the practicality of tobacco laws. There are ongoing debate in the USA about the drawing of age limit to 21 for tobacco sale, however, it is suggested that increasing the awareness of society and in overall, decreasing the usage of tobacco should be of paramount importance [12, 29]. Social sensitivity is important for the prevention of tobacco use and every individual have a responsibility in carrying out this society based program, most especially as it related to prevention of tobacco usage among children and youths.

A higher proportion of smoker respondent who believed the smoking ratio in high school was 6-7 adolescent out of 10, believed that major point were cigarettes are provided to minors were vendor and family, believed that minor access to cigarette was easy, ever witnessed tobacco sale and doing nothing against tobacco sales to minor belonged to the current smokers categories, Also, a lower proportion of respondent who believed imprisonment was the penalty for sale of tobacco to minors belonged to the current smokers category. This finding goes to show that current smokers as compared to other categories believed that tobacco use among minor is prevalent and access is easy, however as compared to other smoking categories were less aware that prison sentence was a part of the law, they also were more aware that minors can access tobacco product through social source such as the family, in addition, current smokers witnessed more sales of tobacco product to minors and did nothing. Non-smokers and highly informed people with regards to tobacco control law intervened more when they witness tobacco sales to minors. In the prevention of smoking among minors, adults should not only be role models for children but they should also warn them about the right behaviors directly. Because of this behavioral difference, two logistic model were conducted.

The results of logistic regression of participant's response against tobacco sales to minor and related factors for current smokers showed that sociodemographic characteristics of participants did not affect the behavior of the person. Only, respondents who believed smoking ratio in high school was 4-5 adolescent out of 10 (aOR: 1.59; 95\% CI: $1.09-2.34$ ) were more likely to give a warning or informing the police or other people as compared to respondents whose perception on the smoking ratio among high school students was 6-7 adolescents out of 10. According to this results of model, we could not explain the witness smokers' reaction to sale of cigarettes with sociodemographic factors and their knowledge on tobacco sale items of the law. It is need the psychosocial behavior theories should be studied on smokers behaviors to understand the causes under their behaviors to strengthen the response of smokers. On the other hand, smoking cessation interventions would change the behaviors of smokers when they witness tobacco sales to minors.

The results of logistic regression of non-smokers' response against tobacco sales to minor were who are from higher educational level, higher economic status, working status and who believed smoking ratio in high school was 4-5 adolescent out of 10 and 2-3 adolescent out of 10 were more likely to give a warning or informing the police or other people as compared to the others. The people from higher socioeconomic status could take more responsibility to monitor the sale of tobacco products to minors. Information and education interventions on tobacco sale to minors would be given to the community, especially to low socioeconomic status in the community. Additional interventions aimed at individual responsibilities are needed for individual smokers about the ban of cigarette sale to children as earlier stated.

\section{Study limitations}

This study has some limitations. Firstly, the study finding is not representative for the whole country. Sampling of cities and the people interviewed were through convenient sampling, as such, the results should be carefully interpreted. Secondly, the average duration of questionnaire completion was approximately 3-4 min, perhaps, due to the time duration some questions were left unanswered by the participants. During data entry, some of these questions were removed and not used in this study.

\section{Conclusions}

Though adults think that tobacco use is a prevalent among minors, tobacco product sales ban is an important subject in tobacco accessibility, most especially at points of sales, unfortunately not enough is known by the adults and they do not take enough responsibility in ensuring the implementation of the tobacco control law as it pertain access to tobacco products by minors. Though Turkey has already made important accomplishments with regards to tobacco control, there is still a need for detailed studies and some interventions are needed to reduce accessibility of minors to tobacco products. 


\section{Abbreviations}

FCTC: Framework Convention on Tobacco Control; GATS: Global Adult Tobacco Survey; GYTS: Global youth tobacco survey; WHO: World Health Organization

\section{Acknowledgements}

Not applicable.

\section{Funding}

This work was supported by the Hacettepe University Scientific Research Projects Coordination Unit.

\section{Availability of data and materials}

Please contact the corresponding author for data requests.

\section{Authors' contributions}

$\mathrm{HO}$ and $\mathrm{NB}$ designed, $\mathrm{HO}$ supervised the study, El and $\mathrm{HU}$ made statisctics analyses, $\mathrm{HO}, \mathrm{NB}, \mathrm{El}$ and $\mathrm{HU}$ wrote the manuscript, the all researchers collected the data and made contributions to the interpretation of results and revised the manuscript. All authors read and approved the final version.

\section{Competing interests}

The authors declare that they have no competing interests. This study was also presented as a poster in European Public Health Conference 2015.

\section{Consent for publication}

Not applicable.

\section{Ethics approval and consent to participate}

All participants provided informed consent before participating in the survey. This study was approved by Non-interventional Clinical Researches Ethics Board of Hacettepe University (GO 13/242-19).

\section{Author details}

${ }^{1}$ Institute of Public Health, University of Hacettepe, Ankara, Turkey. ${ }^{2}$ Yalova Vocational High School, University of Yalova, Yalova, Turkey. ${ }^{3}$ Department of Public Health, Faculty of Medicine, University of Adnan Menderes, Aydın, Turkey. ${ }^{4}$ Department of Public Health, Faculty of Medicine, University of Karadeniz Teknik, Trabzon, Turkey. ${ }^{5}$ Department of Public Health, Faculty of Medicine, University of Osman Gazi, Eskişehir, Turkey. ${ }^{6}$ Department of Public Health, Faculty of Medicine, University of Erciyes, Kayseri, Turkey. ${ }^{7}$ Provincial Directorate of Public Health, Izmir, Turkey. ${ }^{8}$ Department of Public Health, Faculty of Medicine, University of Kirklareli, Krrklareli, Turkey. ${ }^{9}$ Department of Public Health, Faculty of Medicine, University of Ataturk, Erzurum, Turkey.

\section{Received: 28 November 2015 Accepted: 26 November 2016}

\section{Published online: 05 December 2016}

\section{References}

1. World Health Organization (WHO). WHO report on the global tobacco epidemic: The MPOWER package. http://www.who.int/tobacco/mpower/ mpower_report_full_2008.pdf. Accessed 03 Sept 2015.

2. World Health Organization (WHO). WHO Framework Convention on Tobacco Control. Geneva: WHO; 2003.

3. Surgeon General's Report. Preventing tobacco use among youth and young adults. http://www.cdc.gov/tobacco/data_statistics/sgr/2012/index.htm. Accessed 03 Sept 2015.

4. Law No. 5261, For ratification of the WHO framework convention on tobacco control. T.C. Official Gazette, 30 November 2004:25656. http://www. resmigazete.gov.tr/main.aspx?home=http://www.resmigazete.gov.tr/eskiler/ 2004/11/20041130.htm\&main=http://www.resmigazete.gov.tr/eskiler/2004/ 11/20041130.htm. Accessed 03 Sept 2015.

5. Forster JL, Murray DM, Wolfson M, et al. The effects of community policies to reduce youth access to tobacco. Am J Public Health. 1998;88:1193-8.

6. Jason LA, Ji PY, Anes MD, et al. Active enforcement of cigarette control laws in the prevention of cigarette sales to minors. JAMA. 1991;266(3):159-61.

7. Forester $J \mathrm{~L}$, et al. The Effects of Community Policies to Reduce Youth Access to Tobacco. Am J Pub Health. 1998:88:1193. 1197.

8. Lantz MP, Jacobson DP, Warner EK, Wasserman J, Pollack AH, Berson J, Ahlstrom A. Investing in youth tobacco control: a review of smoking prevention and control strategies. Tob Control. 2000;9:47-63.
9. Anon. Tobacco use and usual source of cigarettes among high school students—United States, 1995. MMWR. 1996;45:413-8.

10. Altman DG, Wheelis AY, McFarlane M, et al. The relationship between tobacco access and use among adolescents:a four community study. SocSci Med. 1999:48:759-75.

11. Anon. Accessibility of tobacco products to youths ages 12-17 years_United States, 1989 and 1993. MMWR. 1996;45:125-30.

12. Winickoff JP, McMillen R, Tanski S, Wilson K, Gottlieb M, Crane R. Public support for raising the age of sale for tobacco to 21 in the United States. Tob Control. 2015; tobaccocontrol-2014-052126Published Online First: 20 February 2015. doi:10.1136/tobaccocontrol-2014-052126.

13. Egbe OC, Petersen I, Weitz MA, Asante OK. An exploratory study of the socio-cultural risk influences for cigarette smoking among Southern Nigerian youth. BMC Public Health. 2014;14:1204. Page 2 of 9. http://www. biomedcentral.com/1471-2458/14/1204.

14. Louis Harris and associates. The prostep report on smoking and the family. New York: Louis Harris and Associates Inc; 1992.

15. U.S. Department of Healthandhumanservices. Elders JM, editors. PreventingTobaccoUseAmongYoung People: A Report of theSurgeon General. Washıngton D.C: U.S. Government Printing Office; 1994

16. SE Marcus, SL Emont, RD Corcoran, GA Giovino, JP Pierce, MN Waller, RM Davis. Public attitudes about cigarette smoking: results from the 1990 Smoking Activity Volunteer Executed Survey. Public Health Rep. 1994;109(1):125-34.

17. California department of health services. Tobacco use in California, 1990: a preliminary report documenting the decline of tobacco use. Sacremento: California department of health services; 1991.

18. Centers for Disease Control. Public attitudes regarding limits on public smoking and regulation of tobacco sales and advertising-10 U.S. communities, 1989. Morb Mortal Wkly Rep. 1991;40(21):344-5. 351-3.

19. Law No. 4207, Law on prevention and control of hazards of tobacco products. Article. 4/8, Accepted: 07.11.1996. Available: http://www.mevzuat. gov.tr/Metin.Aspx?MevzuatKod=1.5.4207\&Mevzuatlliski= 0\&sourceXmlSearch=. Accessed on 03 Sept 2015.

20. WHO Regional Office for Europe. Tobacco Control in Prcatice Article 8: Protection from exposure to tobacco smoke. Copenhagen: WHO; 2012.

21. Law No. 5727, BillAmendingtheLaw on Prevention of Hazards of TobaccoProducts. Date of Enactment: 3/1/2008. Available: http://mevzuat. meb.gov.tr/html/22829_4207.html. Accessed on 03 Sept 2015.

22. Republic of Turkey Ministry of Health. Global Adult Tobacco Survey (GATS) 2012. http://www.who.int/tobacco/surveillance/survey/gats/report_tur_2012. pdf?ua=1. Accessed Sept 03, 2015.

23. World HealthOrganization (WHO). WHO global report on trends in tobacco smoking 2000-2025. http://www.who.int/tobacco/publications/surveillance/ reportontrendstobaccosmoking/en/. Accessed 03 Sept 2015.

24. World Health Organization. WHO Framework Convention on Tobacco Control: Guidelines for Implementation. Geneva: World Health Organization; 2011.

25. Ergüder T, Soydal T, Uğurlu M, Çakır B, Warren CW. Tobaccouseamongyouthandrelatedcharacteristics, Turkey. SozPreventiveMed. 2006;51:91-8.

26. US Department of Health and Human Services. Preventing tobacco use among young people. A report of the Surgeon General. Atlanta, Georgia: Public Health Service, Centers for Disease Control and Prevention, Office on Smoking and Health, 1994. (US Government Printing Office Publication No S/N 017-001-00491-0.). Reprinted with corrections, July 1994.

27. Centers for Disease Control. Accessibility of cigarettes to youths aged 12-17 years-United States, 1989. Morb Mortal Wkly Rep. 1992;41(27):485-8.

28. Stead LF, Lancaster T. Interventions for preventing tobacco sales to minors. Cochrane Database Syst Rev. 2005;Issue 1. Art. No.: CD001497. doi: 10.1002/ 14651858.CD001497.pub2.

29. Shari Kessel Schneider, Stephen L Buka, Kim Dash, Jonathan P Winickoff, Lydia O'Donnell. Brief report Community reductions in youth smoking after raising the minimum tobacco sales age to 21. Tob Control. 2015; Published Online First: 12 June 2015. doi:10.1136/tobaccocontrol-2014-052207. 